\title{
The Use of Drosophila melanogaster as a Model Organism to Study the Effect of Bacterial Infection on Host Survival and Metabolism
}

\author{
Jawaher Alasmar ${ }^{1}$, Sara Rashwan ${ }^{1}$
}

Supervised by: Dr. Layla Y. Kamaredine ${ }^{1}$

${ }^{1}$ College of Health Sciences-Biomedical Science Department-Qatar University

\begin{abstract}
Infection-induced metabolic alterations is of great interest to many researchers. Here, we addressed the effect of E.coli, S.enterica, and S.sonnei infection on altering metabolic homeostasis of an infected host using the Drosophila melanogaster (DM) fruit fly model.

Our results revealed that both E.coli and $S$. sonnei were able to colonize the host gut and reduce its life span. Also, these orally infected E.coli and S. sonnei flies exhibited an alteration in the normal mobilization of lipids from the gut to the fat body, an increase in glucose and triglyceride levels, and a down-regulate in the expression of different peptide hormones (AstA, $D H 31$, and $T k$ ) known to regulate metabolic homeostasis in flies. On the contrary, S.enterica was unable to colonize the intestine of host; therefore, the metabolic status of S.enterica infected flies was unaltered.
\end{abstract}

\section{INTRODUCTION}

Large body of evidence have shown that an individual's array of commensal and pathogenic microbes contribute to chronic metabolic diseases such as obesity and diabetes. Therefore understanding different facets of host-pathogen cross-talk is imperative for defining the molecular bases of such metabolic disorders.

The huge similarity between a fruit fly and a mammalian intestine, along with the simplicity of a fly's signaling system as compared to that of mammals, and to its readily available genetic tools, make it a model organism of choice.

Here, we show an effect of S.sonnei, S.enterica and E. coli intestinal pathogenic infections on altering the metabolic status of an infected host.

\section{METHODOLOGY}

1 Drosuphila melanggaster rearing and maintenance

2 Bacterial culture and Drosuphila melanogaster 2 infection

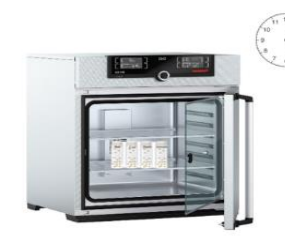

3 Survival assay and colonization of the fly gut

4 Drgan dissection and fluorescence micruscopy

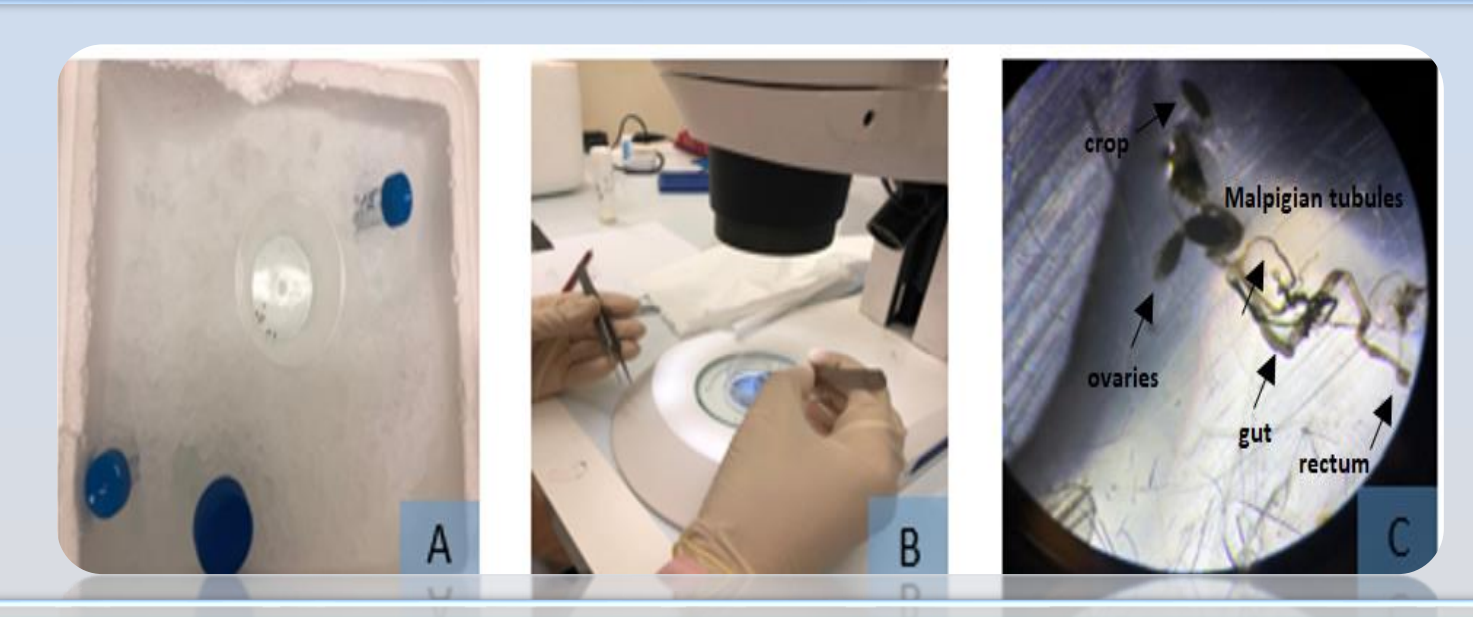

$5 \quad$ Glucose and Triglyceride nutritional assays

6 Metabolic peptide hormone gene expression -Total nucleic acid extraction -DNA removal and RNA extraction -Reverse transcriptase and cDNA synthesis -RT-qPCR

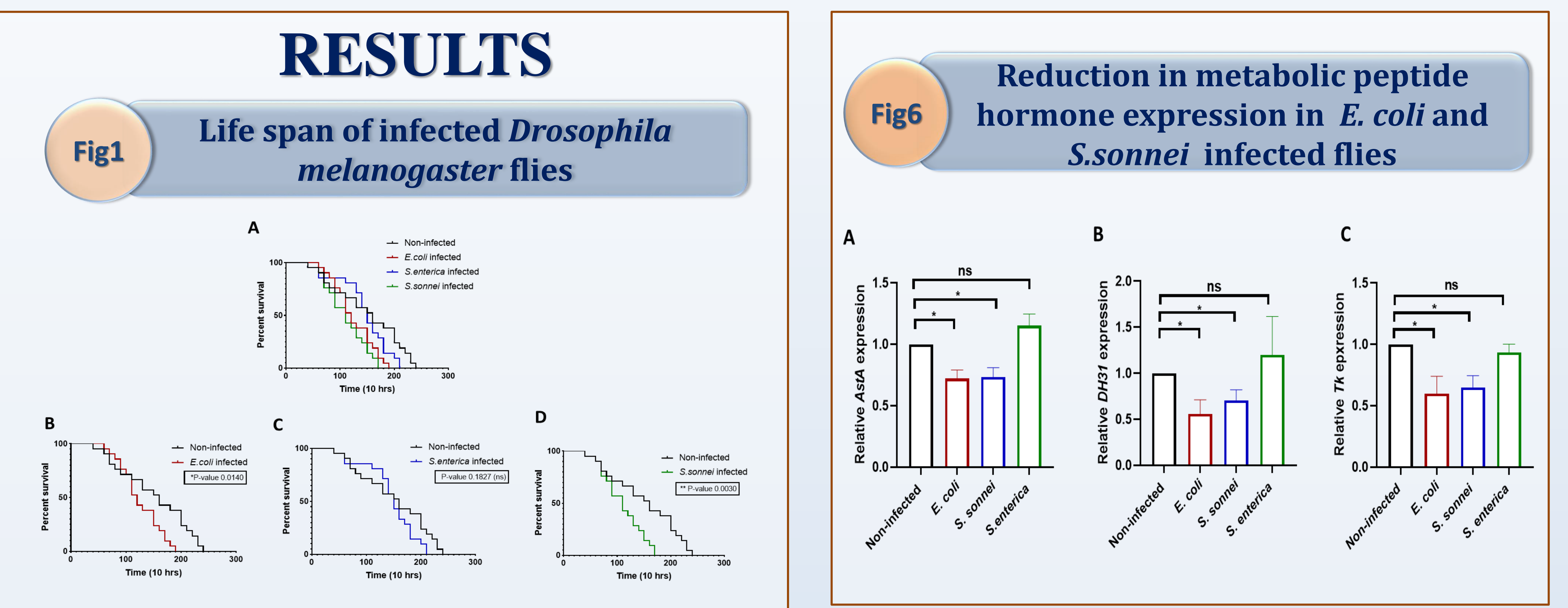

Fig2 Gut colonization of enteric pathogens

\section{CONCLUSIONS}

$\square$ Infection with E.coli and S.sonnei (two gastrointestinal tract disease causing agents)disrupt many metabolic parameters in a host including normal lipid distribution and storage, and systemic glucose and triglyceride levels.

$\square$ This metabolic alteration in E.coli and S.sonnei infected flies is thought to happen through a differential down-regulation in the production and/or secretion of $T k, A s t A$, and DH31 metabolic peptide hormones.

Fig3 Lipid depletion from the fat body of $E$. coli and $S$. sonnei infected flies
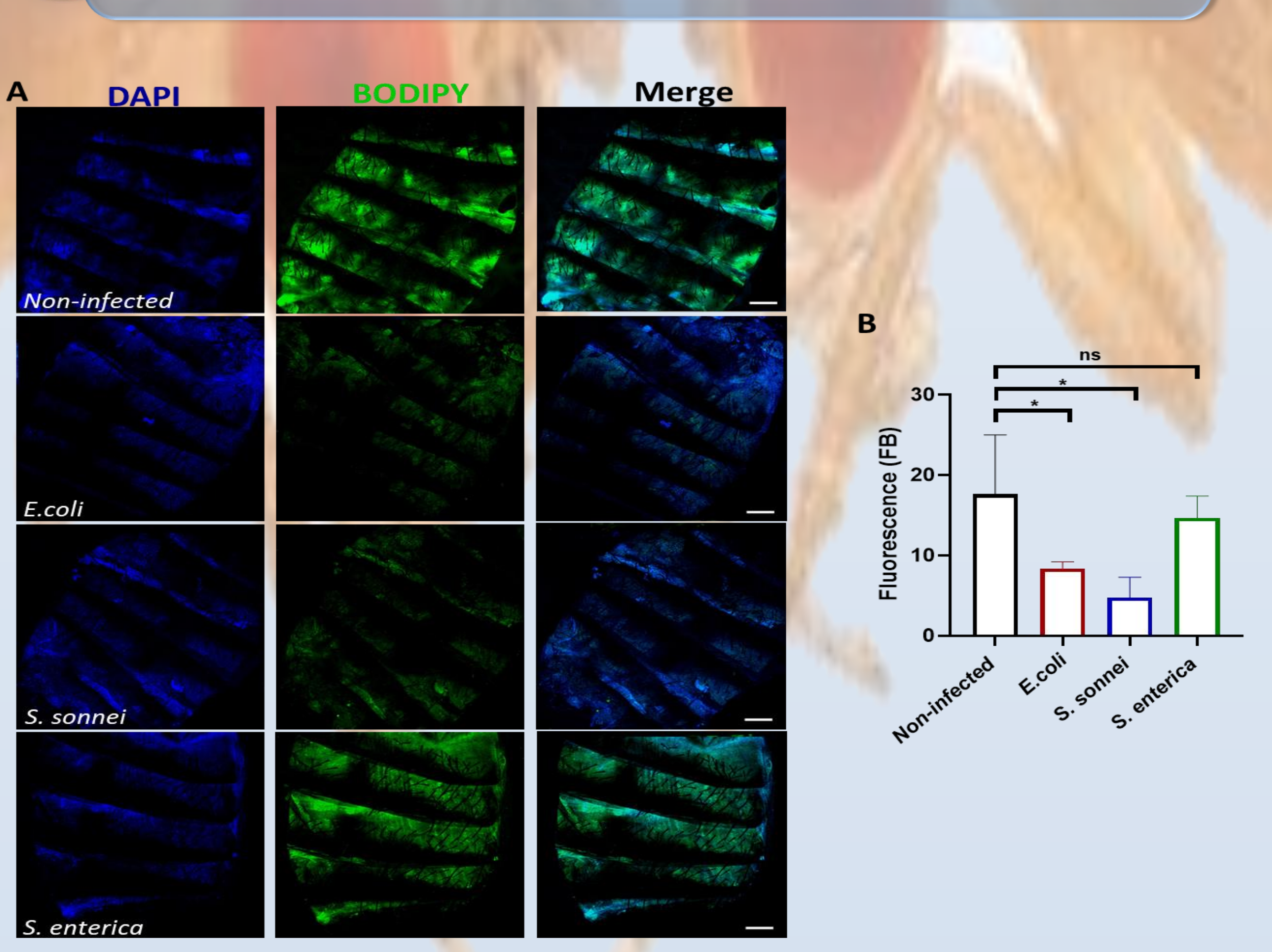

Fig4 Irregular lipid accumulation in the gut
of $E$. coli and S.sonnei infected flies

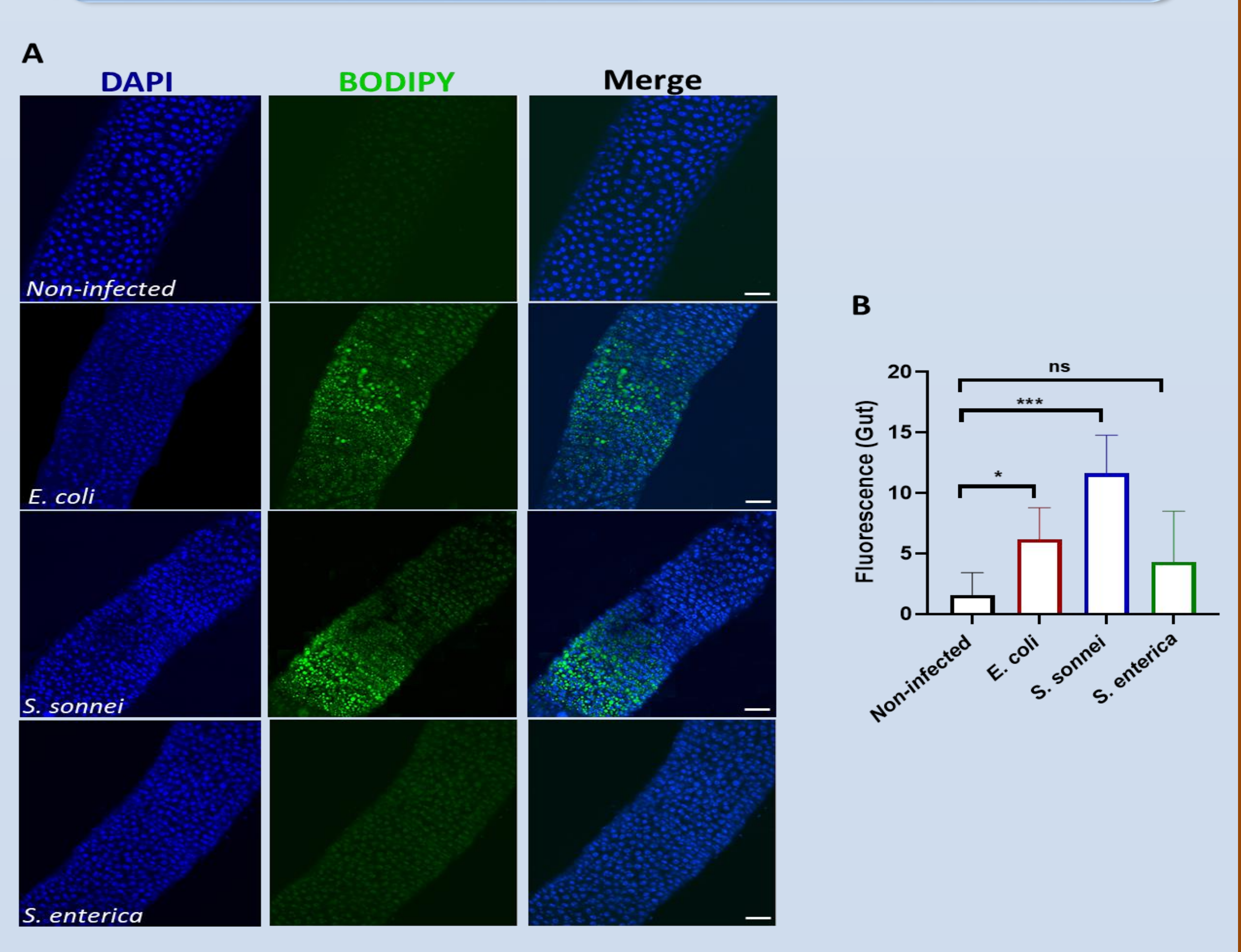

Elevated glucose and triglyceride levels Fig5 in $E$. coli and S.sonnei infected flies

A
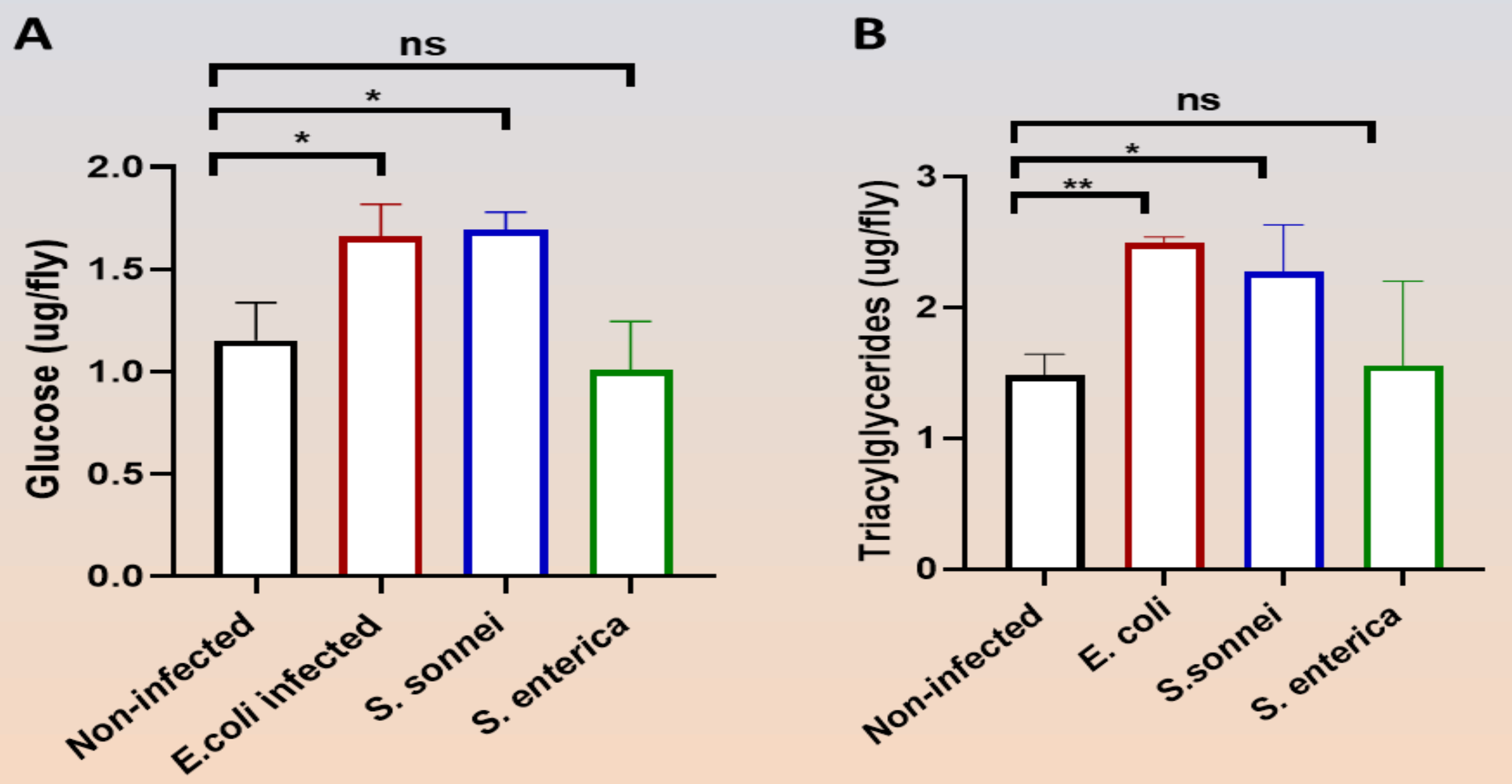

$\square$ Our findings serve as a foundation for future studies to develop profound implications that modulate the pathogenesis of gastrointestinal tract diseases, and open up for promising therapeutic approaches for infection-induced metabolic alterations.

\section{REFRENCES}

- Ayres J.S., and Schneider D.S. (2009). The role of anorexia in resistance and tolerance to infections in Drosophila. PLoS Biol. 7, e1000150 10.1371/journal pbio.1000150

口 Dionne M.S, Pham L. N, Shirasu-Hiza M, and Schneider D. S. (2006). Akt and FOXO dysregulation contribute to infectioninduced wasting in Drosophila. Curr. Biol. 16, 1977-1985. 10.1016/j.cub.2006.08.052.

- Hang S., Purdy A.E., Robins W.P., Wang Z., Mandal M., Chang S., an intestinal pathogen disrupts host insulin signaling and lipid metabolism. Cell Host Microbe 16, 592-604. 10.1016/j.chom.2014.10.006

a Miele L, Giorgio V., Alberelli M. A., De Candia E, Gasbarrini A. and Grieco A. (2015). Impact of gut microbiota on obesity, diabetes, and cardiovascular disease risk. Curr. Cardiol. Rep. 17, 120 10.1007/s11886-015-0671-z

- Subramanian S., Huq S., Yatsunenko T., Haque R., Mahfuz M., Alam M. A., Benezra A., DeStefano J., Meier M. F., Muegge B. D. et al. (2014). Persistent gut microbiota immaturity in malnourished Bangladeshi children. Nature 510, 417-421. 10.1038/nature13421

\section{ACKNOWLEDGEMENTS}

We would like to thank Qatar university, College of Health Sciences, and the Biomedical Science Department for providing us with a special education opportunity that we always desired to have and for facilitating a great environment of learning.

Also, we would like to appreciate the efforts of our graduation project supervisor, assistant professor Dr. Layla Y. Kamareddine for her supporting in providing us with essential instructions needed to complete this work. Mekalanos J.J., and Watnick P.I. (2014). The acetate switch of 